\title{
ON ASYMPTOTIC FORMULAS FOR CERTAIN $q$-SERIES INVOLVING PARTIAL THETA FUNCTIONS
}

\author{
SIHUN JO AND BYUNGCHAN KIM \\ (Communicated by Kathrin Bringmann)
}

ABStRACT. In this article, we investigate the integer sequence $a_{d}(n)$, where for a positive integer $d, a_{d}(n)$ is defined by

$$
\prod_{n=1}^{\infty} \frac{1}{1-q^{n}} \sum_{n=0}^{\infty}(-1)^{n} q^{\left(d n^{2}-(d-2) n\right) / 2}=\sum_{n=0}^{\infty} a_{d}(n) q^{n} .
$$

In particular, we will present a combinatorial model for $a_{d}(n)$ via integer partitions and this will play a crucial role in obtaining an asymptotic formula for $a_{d}(n)$. Moreover, we will show that $p(n)-2 a_{d}(n)$ has an unexpected sign pattern via combinatorial arguments and asymptotic formulas.

\section{INTRODUCTION}

A partial theta function is a sum of the form

$$
\sum_{n=0}^{\infty}(-1)^{n} q^{n(n-1) / 2} x^{n} .
$$

There are many identities involving partial theta functions in Ramanujan's lost notebook 2]. Typically, Ramanujan did not record any proof or motivation for his study of partial theta functions. Thus, we do not know what led Ramanujan to study partial theta functions. However, combinatorially, $q$-series involving partial theta function could be very interesting. For example, G. E. Andrews [4] discussed the following series:

$$
\begin{aligned}
\frac{1}{(q)_{\infty}} \sum_{n=0}^{\infty}(-1)^{n} q^{\left(n^{2}+n\right) / 2} & =1+q^{2}+2 q^{3}+3 q^{4}+4 q^{5}+6 q^{6}+8 q^{7}+12 q^{8}+16 q^{9}+\cdots \\
& =\sum_{n=0}^{\infty} a(n) q^{n}
\end{aligned}
$$

where $(q)_{\infty}=\prod_{n=1}^{\infty}\left(1-q^{n}\right)$ for $|q|<1$. Due to the partial theta function on the left side of (1.1), it is not clear at all that $a(n)$ is positive for all integers $n \geq 2$. A partition of a positive integer $n$ is a weakly decreasing sequence of positive integers

Received by the editors September 28, 2012 and, in revised form, October 1, 2012, January 7, 2013 and September 7, 2013.

2010 Mathematics Subject Classification. Primary 11P82, 11P81.

Key words and phrases. Partial theta function, integer partitions, asymptotic formula.

This work was supported by the Basic Science Research Program through the National Research Foundation of Korea (NRF) funded by the Ministry of Science. 
$\left(\lambda_{1}, \ldots, \lambda_{\ell}\right)$ such that $\lambda_{1}+\cdots+\lambda_{\ell}=n$. We denote by $p(n)$ the number of partitions of $n$. Under the convention that $p(0)=1$, it is well known that

$$
\sum_{n=1}^{\infty} p(n) q^{n}=\frac{1}{(q)_{\infty}}
$$

Since $\frac{1}{(q)_{\infty}}$ appears in (1.1) and $a(n)$ looks positive, it is natural to find a combinatorial explanation for what $a(n)$ counts. Actually, there are several combinatorial interpretations available via integer partitions as we will see later. Once we establish the positivity, the next natural question is how fast does $a(n)$ grow? In a famous work of G. H. Hardy and S. Ramanujan [10, they introduce the circle method to establish an asymptotic formula for the partition function $p(n)$ :

$$
p(n) \sim \frac{1}{4 \sqrt{3} n} \exp \left(\pi \sqrt{\frac{2 n}{3}}\right) .
$$

A modular transformation formula for the generating function for $p(n)$ plays a key role. Unfortunately, many generating functions do not have a modular transformation property, so the Hardy-Ramanujan circle method cannot be applied. However, in a recent work, K. Bringmann and K. Mahlburg 7] introduce a way to get an asymptotic formula by focusing on behavior of the generating function at $q=1$ or by employing Ingham's Tauberian theorem [11. Motivated by recent works of Andrews [4] and Bringmann and Mahlburg [7], the goal of this article is to investigate $a_{d}(n)$, where for a positive integer $d, a_{d}(n)$ is defined by

$$
\frac{1}{(q)_{\infty}} \sum_{n=0}^{\infty}(-1)^{n} q^{\left(d n^{2}-(d-2) n\right) / 2}=\sum_{n=0}^{\infty} a_{d}(n) q^{n} .
$$

In particular, we will present a combinatorial model for $a_{d}(n)$ via integer partitions and will discuss the growth of $a_{d}(n)$. Note that when $d=1, a_{1}(n)=a(n)$. For this case, Andrews 4 proves $a_{1}(n)$ is the number of partitions of $n$ in which the first non-occurence number as a part is odd. To see what $a_{d}(n)$ counts, we need to introduce subpartitions. A partition $\lambda$ of $n$ is a non-increasing sequence of natural numbers whose sum is $n$. Let $\lambda_{1} \geq \lambda_{2} \geq \cdots \geq \lambda_{\ell}$ be a partition. L. Kolitsch 15] introduced the Rogers-Ramanujan subpartitions and established their connection to other partitions. The Rogers-Ramanujan subpartition is the longest sequence satisfying $\lambda_{1}>\lambda_{2}>\cdots>\lambda_{s}$ and $\lambda_{s}>\lambda_{s+1}$ (tail condition), where $\lambda_{i}-\lambda_{j} \geq 2$ for all $i<j \leq s$ (gap condition). By modifying the gap condition, the second author 13. introduced a subpartition with gap $d$, which is defined as the longest sequence satisfying $\lambda_{1}>\lambda_{2}>\cdots>\lambda_{s}$ and $\lambda_{s}>\lambda_{s+1}$, where $\lambda_{i}-\lambda_{j} \geq d$ for all $i<j \leq s$. In a subpartition, we say that $s$ is the length of subpartition. Let us define $p_{e}(n, d)$ $\left(p_{o}(n, d)\right.$, resp. $)$ to be the number of partitions of $n$ that have subpartitions with gap $d$ of even (odd, resp.) lengths. Then, the second author [13] showed that

$$
\sum_{n=0}^{\infty}\left(p_{e}(n, d)-p_{o}(n, d)\right) q^{n}=\frac{1}{(q)_{\infty}}\left(1+2 \sum_{\ell=1}^{\infty}(-1)^{\ell} q^{S_{d, \ell}}\right),
$$

where

$$
S_{d, \ell}=\frac{d \ell^{2}-(d-2) \ell}{2}
$$


In particular, since $p_{e}(n, d)+p_{o}(n, d)=p(n)$, the second author [13] observed that

$$
\sum_{n=0}^{\infty} p_{e}(n, d) q^{n}=\frac{1}{(q)_{\infty}}\left(1+\sum_{\ell=1}^{\infty}(-1)^{\ell} q^{S_{d, \ell}}\right) .
$$

From (1.3) and (1.5), we see that

$$
a_{d}(n)=p_{e}(n, d) .
$$

Combinatorially, it is not hard to see that if $\lambda$ has a subpartition with gap 1 of even length, then the first non-occurence number of the conjugation of $\lambda$ is odd. Moreover, if $s$ is the length of the subpartition with gap 1 in the partition $\lambda$, then $s+1$ is the first non-occurence number as a part in the conjugation of $\lambda$. In this sense, $a_{d}(n)$ is a generalization of Andrews' $a(n)$.

Since every partition of $n$ has a subpartition with gap $d$ of either even length or odd length (in particular, when $d=1$, as the first missing number in a partition of $n$ is either even or odd), we expect that

$$
a_{d}(n) \sim \frac{1}{2} p(n)
$$

as $n$ goes to the infinity. Our first theorem says this is actually true for all positive integers $d$.

Theorem 1.1. For all positive integers $d$,

$$
a_{d}(n) \sim \frac{1}{2} p(n)
$$

To prove Theorem 1.1, we will use Ingham's Tauberian theorem. For employing Ingham's result, it is essential to prove that $a_{d}(n)$ is weakly increasing. To this end, a combinatorial interpretation of $a_{d}(n)$ plays a crucial role.

From Theorem 1.1. we deduce that $p(n)-a_{d}(n)=p_{o}(n, d) \sim \frac{1}{2} p(n)$. Thus, it is surprising if there is a pattern on the sign of $p_{e}(n, d)-p_{o}(n, d)$. Our second theorem manifests this unexpected phenomenon.

Theorem 1.2. For all positive integers $n>2$, we have

$$
\begin{aligned}
p_{e}(n, 1)-p_{o}(n, 1) & >0, \\
(-1)^{n}\left(p_{e}(n, 2)-p_{o}(n, 2)\right) & >0, \\
p_{e}(n, d)-p_{o}(n, d) & <0, \quad \text { for all } d \geq 3 .
\end{aligned}
$$

To prove Theorem 1.1, we will employ $q$-series manipulations and construct injections between two partition classes. We remark that when $d=2$, the generating function is essentially a modular form, and this makes the different pattern.

Our last result is how fast $p_{e}(n, d)-p_{o}(n, d)$ grows. As before, a combinatorial interoperation for the difference is an essential part of the proof.

Theorem 1.3. For all positive integers $d>3$, we have

$$
p_{o}(n, d)-p_{e}(n, d) \sim \frac{(d-2) \pi}{4 \sqrt{6} \sqrt{n}} p(n) \sim \frac{(d-2) \pi}{48 \sqrt{2} n^{\frac{3}{2}}} e^{\pi \sqrt{\frac{2 n}{3}}} .
$$

On the other hand, we obtain that

$$
p_{e}(n, 1)-p_{o}(n, 1) \sim \frac{\pi}{2 \sqrt{6} \sqrt{n}} p(n) \sim \frac{\pi}{24 \sqrt{2} n^{\frac{3}{2}}} e^{\pi \sqrt{\frac{2 n}{3}}},
$$


and

$$
p_{e}(n, 2)-p_{0}(n, 2) \sim \frac{(-1)^{n}}{4}\left(\frac{2}{3 n^{3}}\right)^{\frac{1}{4}} e^{\pi \sqrt{\frac{n}{6}}} .
$$

Remark 1.4. Recently, the second author and E. Kim [14] extended this result and this shows that the asymptotic formula (1.6) holds for the $d=3$ case, too.

When $d=2$, the generating function is essentially a modular form, and thus a more precise result is available. For example, one can find an infinite series representation using I-Bessel functions in a classical work of P. Hagis [9] (note that $p_{e}(n, 2)-p_{o}(n, 2)=S(n)$ in the loc. cit.) and the last asymptotic formula of Theorem 1.3 follows from Hagis' result.

This paper is organized as follows. In Section 2, we give combinatorial arguments for $a_{d}(n)$ or $p_{e}(n, d)-p_{o}(n, d)$, which we will need in the next section. In particular, we prove Theorem 1.2. In Section 3, we obtain asymptotic formulas in Theorems 1.1 and 1.3 .

\section{Combinatorial Results}

In this section, we prove combinatorial lemmas which are essential to derive asymptotic formulas. Before stating results, here we introduce standard $q$-series notation:

$$
\begin{aligned}
(a ; q)_{0} & :=1 \\
(a ; q)_{n} & :=(1-a)(1-a q) \cdots\left(1-a q^{n-1}\right), n \geq 1, \\
(a ; q)_{\infty} & :=\lim _{n \rightarrow \infty}(a ; q)_{n},|q|<1
\end{aligned}
$$

and

$$
\left(a_{1}, a_{2}, \ldots, a_{k} ; q\right)_{\infty}:=\left(a_{1} ; q\right)_{\infty}\left(a_{2} ; q\right)_{\infty} \cdots\left(a_{k} ; q\right)_{\infty} .
$$

Recall that $a_{d}(n)=p_{e}(n, d)$ for all positive integers $n$ and $d$.

Lemma 2.1. $a_{d}(n)$ is weakly increasing in $n$.

Proof. Suppose that $\lambda$ is a partition of $n$ with even subpartition. We split into two cases: (1) The length of subpartition is 0 , and (2) The length of subpartition is even larger than 0 . For the first case, we add a part 1 in the partition $\lambda$. Then, the resulting partition is a partition of $n+1$ where the size of the first two parts is the same, i.e., the length of the subpartition is 0 . For the second case, we increase the largest part of $\lambda$ by 1 . Then, the resulting partition is a partition of $n+1$ and this operation does not affect the length of the subpartition. It is clear that this map is an injection, thus we complete the proof.

Now we turn into the sign of $p_{e}(n, d)-p_{o}(n, d)$. When $d=1$, as shown in [13,

$$
p_{e}(n, 1)>p_{o}(n, 1)
$$

for all integers $n>2$. We also note that, by using the Jacobi triple product formula,

$$
1+\sum_{n=1}^{\infty}\left(p_{e}(n, 2)-p_{o}(n, 2)\right)(-q)^{n}=\left(-q ; q^{2}\right)_{\infty},
$$

which clearly implies the second inequality in Theorem 1.2 
Now we assume that $d \geq 3$. Then we see that

$$
\begin{aligned}
& \sum_{n=0}^{\infty}\left(p_{o}(n, d)-p_{e}(n, d)\right) q^{n} \\
& =\frac{1}{(q)_{\infty}}\left(2 \sum_{n=1}^{\infty}(-1)^{n+1} q^{S_{d, n}}-1\right) \\
& =\frac{1}{(q)_{\infty}}\left(2 \sum_{n=1}^{\infty}(-1)^{n+1} q^{S_{d, n}} \pm \sum_{n=1}^{\infty}(-1)^{n+1} q^{\left(d n^{2}+(d-2) n\right) / 2}-1\right) \\
& =\frac{1}{(q)_{\infty}}\left(-\sum_{n=-\infty}^{\infty}(-1)^{n} q^{S_{d, n}}+\sum_{n=1}^{\infty}(-1)^{n+1} q^{d n(n-1) / 2}\left(q^{n}-q^{(d-1) n}\right)\right) .
\end{aligned}
$$

Recall the Jacobi product formula 12

$$
\sum_{n=-\infty}^{\infty} a^{n(n+1) / 2} b^{n(n-1) / 2}=(-a,-b, a b ; a b)_{\infty} .
$$

We also need the following identity on the difference of two partial theta functions due to A. Berkovich [5]:

$$
\sum_{n=1}^{\infty}(-1)^{n+1} q^{n(n-1) / 2} \frac{x^{n}-y^{n}}{x-y}=(q, q x, q y ; q)_{\infty} \sum_{n=0}^{\infty} \frac{(x y ; q)_{2 n} q^{n}}{(q, q x, q y, x y ; q)_{n}} .
$$

By setting $a=-q$ and $b=-q^{d-1}$ in (2.1) and $q=q^{d}, x=q$, and $y=q^{d-1}$ in (2.2), we deduce that

$$
\begin{aligned}
& \sum_{n=0}^{\infty}\left(p_{o}(n, d)-p_{e}(n, d)\right) q^{n} \\
& =\frac{\left(q, q^{d-1}, q^{d} ; q^{d}\right)_{\infty}}{(q)_{\infty}}\left(\frac{q-q^{d-1}}{(1-q)\left(1-q^{d-1}\right)} \sum_{n=0}^{\infty} \frac{q^{d n}}{\left(q^{d+1}, q^{2 d-1} ; q^{d}\right)_{n}}\left[\begin{array}{c}
2 n \\
n
\end{array}\right]_{q^{d}}-1\right) \\
& =\frac{\left(q, q^{d-1}, q^{d} ; q^{d}\right)_{\infty}}{(q)_{\infty}}\left(\frac{q-q^{d-1}}{(1-q)\left(1-q^{d-1}\right)} \sum_{n=1}^{\infty} \frac{q^{d n}}{\left(q^{d+1}, q^{2 d-1} ; q^{d}\right)_{n}}\left[\begin{array}{c}
2 n \\
n
\end{array}\right]_{q^{d}}+\frac{2 q-1-q^{d}}{(1-q)\left(1-q^{d-1}\right)}\right),
\end{aligned}
$$

where

$$
\left[\begin{array}{c}
2 n \\
n
\end{array}\right]_{q^{d}}=\frac{\left(q^{d} ; q^{d}\right)_{2 n}}{\left(q^{d} ; q^{d}\right)_{n}^{2}}
$$

is the $q$-binomial coefficient which has positive coefficients in $q$. Therefore, it is clear that $p_{o}(n, 3)>p_{e}(n, 3)$ for all integers $n \geq 3$. Now we assume that $d \geq 4$. Since $q$-expansion of

$$
\frac{q-q^{d-1}}{(1-q)\left(1-q^{d-1}\right)}
$$

is non-negative, it is enough to show that $q$-expansion of

$$
\frac{\left(q, q^{d-1}, q^{d} ; q^{d}\right)_{\infty}}{(q)_{\infty}} \frac{2 q-1-q^{d}}{(1-q)\left(1-q^{d-1}\right)}=\frac{\left(q, q^{d-1}, q^{d} ; q^{d}\right)_{\infty}}{(q)_{\infty}}\left(\frac{1}{1-q}-\frac{1}{1-q^{d-1}}-1\right)
$$

is non-negative. Let $A_{d}(n)$ be the number of partitions of $n$ into parts $\not \equiv 0,1, d-1$ $(\bmod d), B_{d}(n)$ be the number of partitions of $n$ into parts $\not \equiv 0,1, d-1(\bmod d)$ except that the number of occurrences of part 1 is not a multiple of $d-1$. Then, we have the following inequality. 
Lemma 2.2. For all integers $n>2$ and $d \geq 4$,

$$
B_{d}(n)>A_{d}(n)
$$

except that $B_{4}(6)=A_{4}(6)$.

The third inequality in Theorem 1.2 will follow immediately with a calculation of $p_{o}(6,4)-p_{e}(6,4)$.

Proof. Let $\mathcal{A}_{d, n}\left(\right.$ resp. $\left.\mathcal{B}_{d, n}\right)$ be the set of partitions counted by $A_{d}(n)\left(\right.$ resp. $\left.B_{d}(n)\right)$. Now we construct a map

$$
\Phi: \mathcal{A}_{d, n} \rightarrow \mathcal{B}_{d, n}
$$

as follows: Suppose that $\lambda \in \mathcal{A}_{d, n}$ and the smallest part of $\lambda$ is $\ell$. If $\ell$ is not a multiple of $d-1$, then replace $\ell$ by $\ell$ copies of 1 . If $\ell$ is a multiple of $d-1$, we replace $\ell$ by $\ell-d$ and $d$ copies of 1 . This is feasible as $\ell$ is larger than $d-1$ if $d-1 \mid \ell$. Since $d$ is not an allowed part, we cannot get a partition with $d$ ones from the first case. Therefore, the map $\Phi$ is injective. Note that $2+1,3+1(1+1+1+1$ for $d=4), 2+2+1$ are in $\mathcal{B}_{d, n}-\Phi\left(\mathcal{A}_{d, n}\right)$. Moreover, for $n \geq 6$, we define $\lambda$ as $k$ copies of 2 plus 3 (resp. 4) copies of 1 if $n$ is odd (resp. even). The partition $\lambda \in \mathcal{B}_{d, n}-\Phi\left(\mathcal{A}_{d, n}\right)$ except for $n=6$ with $d=4$.

Now we show that $R_{d}(n)$ is weakly decreasing, where

$$
\begin{aligned}
\sum_{n=1}^{\infty} R_{d}(n) q^{n} & =\frac{1}{(q)_{\infty}} \sum_{n=1}^{\infty}(-1)^{n+1} q^{d n(n-1) / 2}\left(q^{n}-q^{(d-1) n}\right) \\
& =\frac{\left(q, q^{d-1}, q^{d} ; q^{d}\right)_{\infty}}{(q)_{\infty}} \frac{q-q^{d-1}}{(1-q)\left(1-q^{d-1}\right)} \sum_{n=0}^{\infty} \frac{q^{d n}}{\left(q^{d+1}, q^{2 d-1} ; q^{d}\right)_{n}}\left[\begin{array}{c}
2 n \\
n
\end{array}\right]_{q^{d}}
\end{aligned}
$$

By using standard techniques, we find that $R_{d}(n)$ counts the number of partitions such that

(1) There is no restriction on the parts $\not \equiv 0,1, d-1(\bmod d)$.

(2) If there are $k$ parts $\equiv 0(\bmod d)$, then every part $\equiv 0,1, d-1(\bmod d)$ is between $d$ and $d k+d$ except that the part 1 is allowed.

(3) The number of occurrences of 1 as a part is not a multiple of $d-1$.

Lemma 2.3. $R_{d}(n)$ is weakly increasing for $d>3$.

Proof. Suppose that $d>3$ and $\lambda$ is a partition counted by $R_{d}(n)$. Let $r(\lambda)$ be the number of occurrences of 1 as a part in the partition $\lambda$. If $r(\lambda) \not \equiv-1(\bmod d-1)$, then by adding one more 1 as part, we get a partition counted by $R_{d}(n+1)$. If $r(\lambda) \equiv-1(\bmod d-1)$, then we replace $r$ ones by $d-2$ and $r(\lambda)-d+3$ ones. Since $r(\lambda)-d+3 \equiv 1(\bmod d-1)$, the resulting partition cannot be obtained from the first case. Thus, this process is clearly injective.

\section{Asymptotic Formulas}

By using the combinatorial information from Section 2, we will prove Theorems 1.1 and 1.3. Before starting the proofs, we introduce some definitions. For $2 a, 2 b \in \mathbb{Z}$ 
with $a>0,2 a \equiv 2 b(\bmod 2), d \geq 3$, and $|q|<1$,

$$
\begin{aligned}
F_{a, b}(q) & :=\frac{1}{(q)_{\infty}} \sum_{n=0}^{\infty}(-1)^{n} q^{a n^{2}+b n}=\sum_{n=0}^{\infty} \alpha_{a, b}(n) q^{n}, \\
G(q) & :=\sum_{n=0}^{\infty}\left(p_{e}(n, 1)-p_{o}(n, 1)\right) q^{n}=\frac{1}{(q)_{\infty}}\left(2 \sum_{n=0}^{\infty}(-1)^{n} q^{\left(n^{2}+n\right) / 2}-1\right)=\sum_{n=0}^{\infty} \beta(n) q^{n},
\end{aligned}
$$

and

$$
G_{d}(q):=\sum_{n=1}^{\infty} R_{d}(n) q^{n}=\frac{1}{(q)_{\infty}} \sum_{n=1}^{\infty}(-1)^{n+1} q^{d n(n-1) / 2}\left(q^{n}-q^{(d-1) n}\right) .
$$

The following lemma shows the asymptotic behaviors of the above three functions as $q \rightarrow 1^{-}$.

Lemma 3.1. We have

$$
\begin{aligned}
\lim _{y \rightarrow 0^{+}}\left(e^{-y} ; e^{-y}\right)_{\infty} F_{a, b}\left(e^{-y}\right) & =\frac{1}{2}, \\
\lim _{y \rightarrow 0^{+}}\left(e^{-y} ; e^{-y}\right)_{\infty} \frac{G\left(e^{-y}\right)}{y} & =\frac{1}{2},
\end{aligned}
$$

and

$$
\lim _{y \rightarrow 0^{+}}\left(e^{-y} ; e^{-y}\right)_{\infty} \frac{G_{d}\left(e^{-y}\right)}{y}=\frac{d}{4}-\frac{1}{2}
$$

Proof. By [6, Theorem 1.1], as $y \rightarrow 0^{+}$, we find that

$$
\begin{aligned}
\left(e^{-y} ; e^{-y}\right)_{\infty} F_{a, b}\left(e^{-y}\right) & =\sum_{n=0}^{\infty}(-1)^{n} e^{-\left(a n^{2}+b n\right) y} \\
& =e^{(2 b-a) y / 4} \sum_{n=0}^{N} \frac{E_{2 n} a^{n} y^{n}}{(2 n) ! 2^{2 n+1}} H_{2 n}\left(\frac{(b-a) \sqrt{y}}{2 \sqrt{a}}\right)+O\left(y^{N+1 / 2}\right),
\end{aligned}
$$

where $E_{n}$ is the $n$-th Euler number and $H_{n}(x)$ is the $n$-th Hermite polynomial. Using the fact that $E_{0}=1$ and $H_{0}(x)=1$, we arrive at

$$
\lim _{y \rightarrow 0^{+}}\left(e^{-y} ; e^{-y}\right)_{\infty} F_{a, b}\left(e^{-y}\right)=\frac{1}{2} .
$$

Similarly, we deduce that

$$
\begin{aligned}
\left(e^{-y} ; e^{-y}\right)_{\infty} \frac{G\left(e^{-y}\right)}{y} & =\frac{1}{y}\left(2 \sum_{n=0}^{\infty}(-1)^{n} e^{-\left(n^{2}+n\right) y}-1\right) \\
& =\frac{e^{\frac{y}{4}}-1}{y}+e^{\frac{y}{4}} \sum_{n=1}^{N} \frac{E_{2 n} y^{n-1}}{(2 n) ! 2^{2 n}} H_{2 n}(0)+O\left(y^{N-1 / 2}\right)
\end{aligned}
$$


and

$$
\begin{aligned}
\left(e^{-y}\right. & \left.; e^{-y}\right)_{\infty} \frac{G_{d}\left(e^{-y}\right)}{y} \\
= & -\frac{1}{y}\left(\sum_{n=0}^{\infty}(-1)^{n} e^{-\left(\frac{d n^{2}}{2}+\left(1-\frac{d}{2}\right) n\right) y}-\sum_{n=0}^{\infty}(-1)^{n} e^{-\left(\frac{d n^{2}}{2}+\left(\frac{d}{2}-1\right) n\right) y}\right) \\
= & -\left(\frac{e^{\left(\frac{1}{2}-\frac{3}{8} d\right) y}-e^{\left(\frac{d}{8}-\frac{1}{2}\right) y}}{2 y}\right) \\
& -\sum_{n=1}^{N} \frac{E_{2 n}(d / 2)^{n-1} y^{n-1}}{(2 n) ! 2^{2 n+1}}\left(e^{\left(\frac{1}{2}-\frac{3}{8} d\right) y} H_{2 n}\left((1-d) \sqrt{\frac{y}{2 d}}\right)-e^{\left(\frac{d}{8}-\frac{1}{2}\right) y} H_{2 n}\left(-\sqrt{\frac{y}{2 d}}\right)\right) \\
& +O\left(y^{N-1 / 2}\right) .
\end{aligned}
$$

Using $E_{2}=-1$ and $H_{2}(0)=-2$, we conclude that

$$
\lim _{y \rightarrow 0^{+}}\left(e^{-y} ; e^{-y}\right)_{\infty} \frac{G\left(e^{-y}\right)}{y}=\frac{1}{2} \quad \text { and } \quad \lim _{y \rightarrow 0^{+}}\left(e^{-y} ; e^{-y}\right)_{\infty} \frac{G_{d}\left(e^{-y}\right)}{y}=\frac{d}{4}-\frac{1}{2} .
$$

The following result is a special case of Ingham's Tauberian theorem 11, Theorem 1] as given in [7, Theorem 5.3]. Ingham's Tauberian theorem [11, Theorem 1] enables us to get symptomatic formulas for $\alpha_{a, b}(n), \beta(n)$, and $R_{d}(n)$ from the behavior of the generating functions $F_{a, b}\left(e^{-y}\right), G\left(e^{-y}\right)$, and $G_{d}\left(e^{-y}\right)$ while $y \rightarrow 0^{+}$.

Theorem 3.2 (Ingham's Tauberian theorem (Theorem 1 of [11] and Theorem 5.3 of [7])). Let $f(q)=\sum_{n=1}^{\infty} a(n) q^{n}$ be a power series with real non-negative coefficients and radius of convergence equal to 1 . If there exist $A>0, \lambda, \alpha \in \mathbb{R}$ such that

$$
f(q) \sim \lambda(-\log q)^{\alpha} \exp \left(-\frac{A}{\log q}\right)
$$

as $q \rightarrow 1^{-}$, then

$$
\sum_{n=0}^{N} a(n) \sim \frac{\lambda}{2 \sqrt{\pi}} \frac{A^{\frac{\alpha}{2}-\frac{1}{4}}}{N^{\frac{\alpha}{2}+\frac{1}{4}}} \exp (2 \sqrt{A N})
$$

as $N \rightarrow \infty$.

Since $a_{d}(n)=\alpha_{\frac{d}{2}, 1-\frac{d}{2}}(n)$ and $p_{e}(n, 1)-p_{o}(n, 1)=\beta(n)$, the following theorem immediately implies Theorem 1.1 and the second statement of Theorem 1.3 .

Theorem 3.3. For a positive integer $d$, as $n \rightarrow \infty$, we have

$$
\begin{aligned}
\alpha_{\frac{d}{2}, 1-\frac{d}{2}}(n) & \sim \frac{1}{2} p(n) \sim \frac{1}{4 \sqrt{3} n} e^{\pi \sqrt{\frac{2 n}{3}}}, \\
\beta(n) & \sim \frac{\pi}{2 \sqrt{6} \sqrt{n}} p(n) \sim \frac{\pi}{24 \sqrt{2} n^{\frac{3}{2}}} e^{\pi \sqrt{\frac{2 n}{3}}},
\end{aligned}
$$

and

$$
R_{d}(n) \sim \frac{(d-2) \pi}{4 \sqrt{6} \sqrt{n}} p(n) \sim \frac{(d-2) \pi}{48 \sqrt{2} n^{\frac{3}{2}}} e^{\pi \sqrt{\frac{2 n}{3}}}
$$


Proof. Note that $(1-q) F_{\frac{d}{2}, 1-\frac{d}{2}}(z)$ and $(1-q) G(q)+2 q$ have real non-negative coefficients. We also note that Lemma 2.3 implies that coefficients of $(1-q) G_{d}(z)$ for $d>3$ are also non-negative.

We set $q=e^{-y}$ and define Dedekind's eta function $\eta(\tau)=q^{\frac{1}{24}}(q ; q)_{\infty}$, where $q=e^{2 \pi i \tau}$. Then, we see that $1-q=1-e^{-y}=y-\frac{y^{2}}{2}+\cdots=-\log q+O\left((\log q)^{2}\right)$ as $q \rightarrow 1^{-}$. Moreover, from the transformation formula of $\eta(\tau), \eta\left(-\frac{1}{\tau}\right)=(-i \tau)^{\frac{1}{2}} \eta(\tau)$, we find that

$$
\frac{1}{\left(e^{-y} ; e^{-y}\right)_{\infty}}=\frac{e^{-\frac{1}{24} y}}{\eta\left(\frac{i y}{2 \pi}\right)}=\frac{e^{-\frac{1}{24} y}\left(\frac{y}{2 \pi}\right)^{\frac{1}{2}}}{\eta\left(\frac{2 \pi i}{y}\right)}=\left(\frac{y}{2 \pi}\right)^{\frac{1}{2}} \exp \left(\frac{\pi^{2}}{6 y}\right)(1+O(y)) .
$$

Hence by Lemma 3.1, we see that

$$
\begin{aligned}
(1-q) F_{\frac{d}{2}, 1-\frac{d}{2}}(z) & \sim \frac{1}{2 \sqrt{2 \pi}}(-\log q)^{\frac{3}{2}} \exp \left(-\frac{\pi^{2} / 6}{\log q}\right), \\
(1-q) G(q)+2 q & \sim \frac{1}{2 \sqrt{2 \pi}}(-\log q)^{\frac{5}{2}} \exp \left(-\frac{\pi^{2} / 6}{\log q}\right)
\end{aligned}
$$

and for $d>3$,

$$
(1-q) G_{d}(q) \sim\left(\frac{d}{4}-\frac{1}{2}\right) \frac{1}{\sqrt{2 \pi}}(-\log q)^{\frac{5}{2}} \exp \left(-\frac{\pi^{2} / 6}{\log q}\right) .
$$

Employing Ingham's Tauberian theorem, we arrive at

$$
\begin{aligned}
& 1+\sum_{k=1}^{n}\left(\alpha_{\frac{d}{2}, 1-\frac{d}{2}}(k)-\alpha_{\frac{d}{2}, 1-\frac{d}{2}}(k-1)\right)=\alpha_{\frac{d}{2}, 1-\frac{d}{2}}(n) \sim \frac{1}{8 \sqrt{3} n} \exp \left(\pi \sqrt{\frac{2 n}{3}}\right) \sim \frac{1}{2} p(n), \\
& 3+\sum_{k=1}^{n}(\beta(k)-\beta(k-1))=2+\beta(n) \sim \frac{\pi}{24 \sqrt{2} n^{\frac{3}{2}}} \exp \left(\pi \sqrt{\frac{2 n}{3}}\right) \sim \frac{\pi}{2 \sqrt{6} \sqrt{n}} p(n),
\end{aligned}
$$

and for $d>3$,

$$
1+\sum_{k=2}^{n}\left(R_{d}(k)-R_{d}(k-1)\right)=R_{d}(n) \sim \frac{(d-2) \pi}{48 \sqrt{2} n^{\frac{3}{2}}} \exp \left(\pi \sqrt{\frac{2 n}{3}}\right) \sim \frac{(d-2) \pi}{4 \sqrt{6} \sqrt{n}} p(n) .
$$

To prove the first statement of Theorem 1.3, we first need the following lemma.

Lemma 3.4. For $d>3$, let

$$
\frac{\left(q, q^{d-1}, q^{d} ; q^{d}\right)_{\infty}}{(q)_{\infty}}=\prod_{n=1}^{\infty}\left(1-q^{n}\right)^{-r_{d, n}}=\sum_{n=0}^{\infty} r_{d}(n) q^{n} .
$$

Then as $n \rightarrow \infty$,

$$
r_{d}(n)=C_{d} n^{-\frac{3}{4}} \exp \left(\pi \sqrt{1-\frac{3}{d}} \sqrt{\frac{2 n}{3}}\right)\left(1+O\left(n^{-\frac{1}{4}+\epsilon}\right)\right),
$$

where $\epsilon$ is an arbitrary real number,

$$
C_{d}=\exp \left(\log \left(2 \pi / d^{\frac{3}{2}}\right)+\sum_{m=1}^{\infty} \frac{(-1)^{m}(2 \pi)^{2 m} B_{2 m}}{2 m(2 m) ! d^{2 m}}\right) \frac{(1-3 / d)^{\frac{1}{4}}}{2^{\frac{5}{4}} 3^{\frac{1}{4}}},
$$

and $B_{2 m}$ is the $2 m$-th Bernoulli number. 
Actually, the generating function of $r_{d}(n)$ is essentially a modular form of weight 0 , thus we could get an asymptotic formula from this fact. (For example, look at [17] and [8].) However, here we will use Meinadus' result [16] for sticking to the theme of the paper from which we derive asymptotic formulas by concentrating on the behavior of generating function near $q=1$. For the statement of Meinadus' result, we follow Andrews' exposition from his book [1, Chapter 6].

Theorem 3.5 (Meinadus' asymptotic formula). Let

$$
f(y)=\prod_{n=1}^{\infty}\left(1-q^{n}\right)^{-a_{n}}=1+\sum_{n=1}^{\infty} a(n) q^{n},
$$

where $q=e^{-y},|q|<1$ and $a_{n} \geq 0$. Suppose that

$$
D(s)=\sum_{n=1}^{\infty} \frac{a_{n}}{n^{s}} \quad(s=\sigma+i t)
$$

satisfies certain conditions (for details, see [16] or [1]) with a simple pole at $s=\alpha$ with residue $A$. Then as $n \rightarrow \infty$,

$$
a(n)=C n^{\kappa} \exp \left(n^{\alpha /(\alpha+1)}\left(1+\frac{1}{\alpha}\right)(A \Gamma(\alpha+1) \zeta(\alpha+1))^{1 /(\alpha+1)}\right)\left(1+O\left(n^{-\kappa_{1}}\right)\right),
$$

where $\zeta(s)=\sum_{m=1}^{\infty} m^{-s}$ is the Riemann zeta function, and

$$
\begin{aligned}
C & =e^{D^{\prime}(0)}(2 \pi(1+\alpha))^{-\frac{1}{2}}(A \Gamma(\alpha+1) \zeta(\alpha+1))^{(1-2 D(0)) /(2+2 \alpha)}, \\
\kappa & =\frac{D(0)-1-\frac{1}{2} \alpha}{1+\alpha} \\
\kappa_{1} & =\frac{\alpha}{\alpha+1} \min \left(\frac{C_{0}}{\alpha}-\frac{\delta}{4}, \frac{1}{2}-\delta\right), \text { for an arbitrary real number } \delta .
\end{aligned}
$$

Since the proof of Lemma 3.4 is immediate from Meinadus' asymptotic formula, we omit it. We are now ready to prove the first asymptotic formula of Theorem 1.3 .

Proof of the first asymptotic formula of Theorem 1.3. If $d>3$, then

$\sum_{n=0}^{\infty}\left(p_{o}(n, d)-p_{e}(n, d)\right) q^{n}=G_{d}(q)-\frac{\left(q, q^{d-1}, q^{d} ; q^{d}\right)_{\infty}}{(q)_{\infty}}=\sum_{n=1}^{\infty} R_{d}(n) q^{n}-\sum_{n=0}^{\infty} r_{d}(n) q^{n}$.

By Theorem 3.3 and Lemma 3.4. we see that $r_{d}(n)=o\left(R_{d}(n)\right)$ as $n \rightarrow \infty$. Hence, we deduce that

$$
R_{d}(n)-r_{d}(n) \sim R_{d}(n) \sim \frac{(d-2) \pi}{4 \sqrt{6} \sqrt{n}} p(n) \sim \frac{(d-2) \pi}{48 \sqrt{2} n^{\frac{3}{2}}} e^{\pi \sqrt{\frac{2 n}{3}}},
$$

as $n \rightarrow \infty$.

\section{ACKNOWLEDGMENTS}

The authors thank George Andrews, Bruce Berndt, and Kathrin Bringmann for valuable comments on an earlier version of this paper. The authors also appreciate the reviewer for the careful reading and helpful comments, which greatly improved the exposition of this article. 


\section{REFERENCES}

[1] George E. Andrews, The theory of partitions, Cambridge Mathematical Library, Cambridge University Press, Cambridge, 1998. Reprint of the 1976 original. MR.1634067 (99c:11126)

[2] G. E. Andrews, Ramanujan's lost notebook. I. partial theta functions, Adv. in Math. 41 (1981), 137-172.

[3] George E. Andrews, The theory of partitions, Cambridge Mathematical Library, Cambridge University Press, Cambridge, 1998. Reprint of the 1976 original. MR.1634067 (99c:11126)

[4] George E. Andrews, Concave compositions, Electron. J. Combin. 18 (2011), no. 2, Paper 6, 13. MR2795783 (2012e:05039)

[5] A. Berkovich, On the difference of partial theta functions, preprint.

[6] Bruce C. Berndt and Byungchan Kim, Asymptotic expansions of certain partial theta functions, Proc. Amer. Math. Soc. 139 (2011), no. 11, 3779-3788, DOI 10.1090/S0002-9939-201111062-1. MR:2823024 (2012h:11067)

[7] Kathrin Bringmann and Karl Mahlburg, Asymptotic inequalities for positive crank and rank moments, Trans. Amer. Math. Soc. 366 (2014), no. 2, 1073-1094, DOI 10.1090/S0002-99472013-05945-4. MR3130326

[8] Kathrin Bringmann and Ken Ono, Coefficients of harmonic Maass forms, Partitions, qseries, and modular forms, Dev. Math., vol. 23, Springer, New York, 2012, pp. 23-38, DOI 10.1007/978-1-4614-0028-8_3. MR3051180

[9] Peter Hagis Jr., On the partitions of an integer into distinct odd summands, Amer. J. Math. 87 (1965), 867-873. MR0188182 (32 \#5621)

[10] G. H. Hardy, Asymptotic Formulaae in Combinatory Analysis, Proc. London Math. Soc. S2-17, no. 1, 75, DOI 10.1112/plms/s2-17.1.75. MR.1575586

[11] A. E. Ingham, A Tauberian theorem for partitions, Ann. of Math. (2) 42 (1941), 1075-1090. MR.0005522(3,166a)

[12] C. C. J. Jacobi, Fundamenta nova theoriae functionum ellipticarum. Regiomonti, fratrum Bornträger (reprinted in Gesammelte Werke, Vol. 1, pp. 49-239; Reimer, Berlin, 1881) (1829)

[13] Byungchan Kim, On the subpartitions of the ordinary partitions, Ramanujan J. 23 (2010), no. 1-3, 159-167, DOI 10.1007/s11139-009-9202-0. MR2739209(2011j:11196)

[14] Byungchan Kim and Eunmi Kim, On the subpartitions of the ordinary partitions, II, Electron. J. Combin. 21 (2014), no. 4, Paper 4.21, 11. MR3292258

[15] Louis W. Kolitsch, Rogers-Ramanujan subpartitions and their connections to other partitions, Ramanujan J. 16 (2008), no. 2, 163-167, DOI 10.1007/s11139-007-9105-x. MR2424918 (2009f:11129)

[16] Günter Meinardus, Asymptotische Aussagen über Partitionen (German), Math. Z. 59 (1954), 388-398. MR0062781 (16,17e)

[17] Hans Rademacher, The Fourier Coefficients of the Modular Invariant J( $\tau)$, Amer. J. Math. 60 (1938), no. 2, 501-512, DOI 10.2307/2371313. MR1507331

Department of Mathematics, Yonsei University, Seoul 120-749, Republic of Korea Current address: School of Mathematics, Korea Institute for Advanced Study, 85 Hoegiro

Dongdaemun-Gu Seoul 130-722, Republic of Korea

E-mail address: topspace@kias.re.kr

School of Liberal Arts and Institute of Convergence Fundamental Studies, Seoul National University of Science and Technology, 232 Gongneung-Ro, Nowongu, Seoul 139-743, Republic of KoreA

E-mail address: bkim4@seoultech.ac.kr 